\title{
New nonlinear dielectric materials: Linear electrorheological fluids under the influence of electrostriction
}

\author{
J. P. Huang \\ Department of Physics, The Chinese University of Hong Kong, Shatin, NT, Hong Kong, and \\ Max-Planck-Institut für Polymerforschung, Ackermannweg 10, 55128, Mainz, Germany
}

\begin{abstract}
The usual approach to the development of new nonlinear dielectric materials focuses on the search for materials in which the components possess an inherently large nonlinear dielectric response. In contrast, based on thermodynamics, we have presented a first-principles approach to obtain the electrostriction-induced effective third-order nonlinear susceptibility for the electrorheological (ER) fluids in which the components have inherent linear, rather than nonlinear, responses. In detail, this kind of nonlinear susceptibility is in general of about the same order of magnitude as the compressibility of the linear ER fluid at constant pressure. Moreover, our approach has been demonstrated in excellent agreement with a different statistical method. Thus, such linear ER fluids can serve as a new nonlinear dielectric material. PACS: 42.65.An, 83.80.Gv, 42.70.Nq
\end{abstract}


Nonlinear materials with large values of the third-order nonlinear susceptibility $\chi[1]$ are in great need in industrial applications, such as, nonlinear optical switching devices for use in photonics and real-time coherent optical signal processors, and exploiting new type of nonlinear dielectric materials for use in electronic and microwave components and sensor windows. It is usually believed that the effective nonlinear dielectric response can appear in the composite material, in which at least one component should possess an inherent nonlinear response. Thus, the common way to develop new nonlinear dielectric materials is to seek for materials in which the components possess an inherently large nonlinear response [2]. In contrast, by using a first-principles approach based on thermodynamics, we shall present a quite different method to obtain the nonlinear dielectric response from the electrorheological (ER) fluids in which the components possess inherent linear responses only (namely, linear ER fluids), under the influence of electrostriction. Thus, such linear ER fluids can also serve as a nonlinear dielectric material because the effective third-order nonlinear susceptibility can be induced due to the electrostriction effect. For clarity, it is worth noting that the above-mentioned linear ER fluids represent the ER fluids whose dielectric constants are independent of the external electric field.

When an ER fluid [3-7] is subjected to a strong external field, elongated chains or columns of polarizable dielectric particles (e.g., titanium particles) form immediately parallel to the field due to the anisotropic long-range particle interaction inside the liquid carrier (e.g., silicone or corn oil). Because of this sort of rapid field-induced aggregation, recently ER fluids have received much attention [4-7] in both scientific research and industrial applications. For instance, ER fluids were also proposed as a method of constructing shock absorbers on magnetically levitated trains.

Let us start by considering what electrostriction effect is for ER fluids. In the presence of an inhomogeneous electric field $\mathbf{E}$, it is known that a translational force $\mathbf{F}_{t}$ exerts on a particle, which is given by

$$
\mathbf{F}_{t}=\alpha \mathbf{E} \cdot \nabla \mathbf{E},
$$


where $\alpha$ represents the polarizability of the particle. Therefore, an inhomogeneous field acting on an ER fluid causes a particle concentration gradient with high concentrations at high field strengths. Next, if the ER fluid is situated partially in a strong external electric field at constant pressure, the density of the ER fluid in the field will increase accordingly due to the interaction between the induced dipole moment inside the particles and the electric field, which in turn yields an increase in the effective dielectric constant. This effect is called electrostriction. In fact, the phenomenon of electrostriction has been extensively studied, e.g. for dipolar fluids [8], near-critical sulfur hexafluoride in miscrogravity [9], ferroelectric liquidcrystalline elastomers [10], an all-organic composite consisting of polyvinylidene fluoride trifluoroethylene copolymer matrix and copper-phthalocyanine particles [11]. Regarding the ER system, one [12] studied the electrostriction of solid ER composites in an attempt to apply them in sensing shear stresses and strains in active damping of vibrations due to the high sensitivity of ER composites to shear electrostriction. To the best of our knowledge, there is neither theoretical nor experimental research which treats the electrostriction effect of ER fluids. In this paper, based on thermodynamics we shall present a first-principles approach to derive the electrostriction-induced effective nonlinear third-order susceptibility $\chi$ of the linear ER fluids.

For investigating the electrostriction effect, take the experimental situation as follows: There is a capacitor with volume $V_{c}$, in which the electric field and the dielectric displacement are denoted by $E_{c}$ and $D_{c}$, respectively. Both of them should satisfy the usual electrostatic equations, namely

$$
\begin{gathered}
\nabla \cdot \mathbf{D}_{c}=0 \\
\nabla \times \mathbf{E}_{c}=0
\end{gathered}
$$

Here Eq. (3) implies that the electric field $\mathbf{E}_{c}$ can be expressed as the gradient of a potential $\phi$

$$
\mathbf{E}_{c}=-\nabla \phi
$$


Under the appropriate boundary condition, the inhomogeneous ER fluid (in the capacitor) can be represented as a region of volume $V_{c}$, surrounded by surface $S_{\mathrm{s}}$. Such kind of boundary condition is

$$
\phi=-\mathbf{E} \cdot \mathbf{R} \text { on } S_{s}
$$

which, if the ER fluid within $V_{c}$ were uniform, would give rise to an electric field which is identical to $\mathbf{E}$ everywhere within $V_{c}$. As a matter of fact, even in an inhomogeneous ER fluid with this boundary condition, the volume average of the electric field $\left\langle\mathbf{E}_{\mathbf{c}}\right\rangle$ within $V_{c}$ still equals that of the external field $\langle\mathbf{E}\rangle$, i.e.

$$
\left\langle\mathbf{E}_{\mathbf{c}}\right\rangle=\frac{1}{V_{c}} \int \mathbf{E}_{c}(\mathbf{R}) \mathrm{d}^{3} r=\langle\mathbf{E}\rangle
$$

It is worth noting that in this case there is no applied field outside the capacitor. Also, the whole ER fluid with volume $V$ is situated both inside and outside the capacitor at a constant pressure $p$.

In the presence of the inhomogeneous external electric field $\mathbf{E}$, the effective linear dielectric constant $\epsilon_{e}$ and effective third-order nonlinear susceptibility $\chi$ for the ER fluid inside the capacitor are defined as

$$
\left\langle\mathbf{D}_{c}\right\rangle=\epsilon_{e}\langle\mathbf{E}\rangle+4 \pi \chi|\langle\mathbf{E}\rangle|^{2}\langle\mathbf{E}\rangle
$$

where $\langle\cdots\rangle$ denotes the volume average of $\cdots$. A similar definition [13] was used for a composite material which is subjected to a homogeneous external electric field. In view of the real quantities under consideration, Eq. (7) can be rewritten as

$$
\left\langle\mathbf{D}_{c}\right\rangle=\epsilon_{e}\langle\mathbf{E}\rangle+4 \pi \chi\langle\mathbf{E}\rangle^{2}\langle\mathbf{E}\rangle \text {. }
$$

On the other hand, based on thermodynamics the effective dielectric constant $\epsilon_{E}$ including the incremental part due to the electrostriction is defined as

$$
\epsilon_{E} \equiv\left(\frac{\partial\left\langle\mathbf{D}_{c}\right\rangle}{\partial\langle\mathbf{E}\rangle}\right)_{T, p}=\left(\frac{\partial\left\langle\mathbf{D}_{c}\right\rangle}{\partial\langle\mathbf{E}\rangle}\right)_{T, \rho}+\int f(d)\left(\frac{\partial\left\langle\mathbf{D}_{c}\right\rangle}{\partial \rho(d)}\right)_{T,\langle\mathbf{E}\rangle}\left(\frac{\partial \rho(d)}{\partial\langle\mathbf{E}\rangle}\right)_{T, p} \mathrm{~d} d,
$$


where $\rho(d)$ stands for the density of the particles with diameter $d$, and $T$ temperature. Here $\left(\frac{\partial\left\langle\mathbf{D}_{c}\right\rangle}{\partial\langle\mathbf{E}\rangle}\right)_{T, \rho}$ corresponds to the effective linear dielectric constant, namely $\epsilon_{e}$. In Eq. $(9), f(d)$ denotes a specific size distribution which exists in real ER fluids [14], e.g. the lognormal distribution $f(d)=\frac{1}{\sqrt{2 \pi} \sigma d} \exp \left[-\frac{\ln ^{2}(d / \delta)}{2 \sigma^{2}}\right]$, where $\sigma$ is the standard deviation and $\delta$ the median diameter.

Accordingly, the incremental dielectric constant due to the electrostriction [the last term of Eq. (9)] is equivalent to $12 \pi \chi\langle\mathbf{E}\rangle^{2}$. That is,

$$
12 \pi \chi\langle\mathbf{E}\rangle^{2}=\int f(d)\left(\frac{\partial\left\langle\mathbf{D}_{c}\right\rangle}{\partial \rho(d)}\right)_{T,\langle\mathbf{E}\rangle}\left(\frac{\partial \rho(d)}{\partial\langle\mathbf{E}\rangle}\right)_{T, p} \mathrm{~d} d .
$$

Let us take one step forward to rewrite Eq. (10) as

$$
\chi\langle\mathbf{E}\rangle^{2}=\frac{1}{12 \pi} \int f(d)\langle\mathbf{E}\rangle\left(\frac{\partial \epsilon_{e}}{\partial \rho(d)}\right)_{T,\langle\mathbf{E}\rangle}\left(\frac{\partial \rho(d)}{\partial\langle\mathbf{E}\rangle}\right)_{T, p} \mathrm{~d} d,
$$

The differential increase of the density inside the capacitor $\mathrm{d} \rho(d)$ corresponds to the increase in mass equal to $V_{c} \mathrm{~d} \rho(d)$. Naturally, this increase in mass is equal to a decrease in mass outside the capacitor, which is given by $-\rho(d) \mathrm{d}\left(V-V_{c}\right)=-\rho(d) \mathrm{d} V$, so that $\mathrm{d} \rho(d)=$ $-\left[\rho(d) / V_{c}\right] \mathrm{d} V$. Consequently, we may rewrite Eq. (11) as

$$
\chi\langle\mathbf{E}\rangle^{2}=-\frac{1}{12 \pi} \int f(d)\langle\mathbf{E}\rangle \frac{\rho(d)}{V_{c}}\left(\frac{\partial \epsilon_{e}}{\partial \rho(d)}\right)_{T,\langle\mathbf{E}\rangle}\left(\frac{\partial V}{\partial\langle\mathbf{E}\rangle}\right)_{T, p} \mathrm{~d} d .
$$

Next, we can obtain $\left(\frac{\partial V}{\partial\langle\mathbf{E}\rangle}\right)_{T, p}$ by using the differential of the free energy $\mathrm{d} F$

$$
\mathrm{d} F=-p \mathrm{~d} V-S \mathrm{~d} T+\frac{V_{c}}{4 \pi}\langle\mathbf{E}\rangle \mathrm{d}\left\langle\mathbf{D}_{c}\right\rangle
$$

where $S$ denotes the entropy. In view of the transformed free enthalpy $G$

$$
G=F+p V-\frac{V_{c}}{4 \pi}\langle\mathbf{E}\rangle\left\langle\mathbf{D}_{c}\right\rangle
$$

the differential of $G$ admits the form

$$
\mathrm{d} G=-S \mathrm{~d} T+V \mathrm{~d} p-\frac{V_{c}}{4 \pi}\left\langle\mathbf{D}_{c}\right\rangle \mathrm{d}\langle\mathbf{E}\rangle
$$

Based on this equation, we obtain 


$$
\left(\frac{\partial V}{\partial\langle\mathbf{E}\rangle}\right)_{T, p}=-\frac{V_{c}\langle\mathbf{E}\rangle}{4 \pi}\left(\frac{\partial \epsilon_{e}}{\partial p}\right)_{T,\langle\mathbf{E}\rangle} .
$$

Then, the substitution of Eq. (16) to Eq. (12) yields

$$
\chi\langle\mathbf{E}\rangle^{2}=\frac{1}{48 \pi^{2}} \int f(d)\langle\mathbf{E}\rangle^{2} \rho(d)\left(\frac{\partial \epsilon_{e}}{\partial \rho(d)}\right)_{T,\langle\mathbf{E}\rangle}\left(\frac{\partial \epsilon_{e}}{\partial p}\right)_{T,\langle\mathbf{E}\rangle} \mathrm{d} d .
$$

Now let us use

$$
\left(\frac{\partial \epsilon_{e}}{\partial p}\right)_{T,\langle\mathbf{E}\rangle}=\beta \rho(d)\left(\frac{\partial \epsilon_{e}}{\partial \rho(d)}\right)_{T}
$$

where

$$
\beta=-\frac{1}{V}\left(\frac{\partial V}{\partial p}\right)_{T}
$$

denotes the compressibility in the absence of the external electric field. For deriving Eq. (18), we have neglected the terms which depends on $\langle\mathbf{E}\rangle$ because they lead to terms in powers of $\langle\mathbf{E}\rangle$ higher than the second in Eq. (17). In the light of the same approximation, the substitution of Eq. (18) into Eq. (17) leads to

$$
\chi\langle\mathbf{E}\rangle^{2}=\frac{1}{48 \pi^{2}} \int f(d)\langle\mathbf{E}\rangle^{2} \beta \rho(d)^{2}\left(\frac{\partial \epsilon_{e}}{\partial \rho(d)}\right)_{T}^{2} \mathrm{~d} d .
$$

So far, the effective third-order nonlinear susceptibility $\chi$ of the ER fluid is given by

$$
\chi=\frac{\beta}{48 \pi^{2}} \int f(d) \rho(d)^{2}\left(\frac{\partial \epsilon_{e}}{\partial \rho(d)}\right)_{T}^{2} \mathrm{~d} d .
$$

For determining the effective linear dielectric constant $\epsilon_{e}$, we can resort to the anisotropic Maxwell-Garnett theory, namely

$$
\frac{g_{L}\left(\epsilon_{e}-\epsilon_{2}\right)}{\epsilon_{2}+g_{L}\left(\epsilon_{e}-\epsilon_{2}\right)}=\frac{4 \pi}{3} \int f(d) \frac{\rho(d)}{m(d)} \alpha(d) \mathrm{d} d
$$

where $m(d)(\alpha(d))$ denotes the mass (polarizability) of the individual particle with diameter $d$, and $\epsilon_{2}$ the dielectric constant of the carrier liquid. It is known that in the presence of an electric field, the particle chain can be formed in the direction of the field, and thus the structural anisotropy should appear inside this ER fluid. Accordingly, in Eq. (22) $g_{L}$ 
$\left(g_{L} \geq 1 / 3\right)$ is the local field factor in the longitudinal field case, which was measured by using computer simulations [15], satisfying the sum rule $g_{L}+2 g_{T}=1$ [16]. Here $g_{T}$ represents the local field factor in the transverse field case. As $g_{L}=1 / 3$, the usual Clausius-Mossotti equation recovers, which is valid for an isotropic system. In fact, the degree of anisotropy of the present system is measured by how $g_{L}$ is deviated from $1 / 3$.

Eq. (21) is the main result of the present paper. In detail, the electrostriction-induced third-order nonlinear susceptibility $\chi$ can be expressed in terms of the size distribution function and density of the particles, the effective linear dielectric constant, etc. In particular, it is apparent to see that at constant pressure $\chi$ is proportional to the compressibility of the ER fluids of interest. More precisely, $\chi$ is of about the same order of magnitude as the compressibility, which can be readily measured in experiments. Let us compute $\chi$ for a real example of an ER fluid by Klingenberg et al. [17]. In detail, this monodisperse ER fluid contains hollow silica spherical particles embedded in a corn oil. The parameters obtained from the experiment: diameter of the particles $95 \mu m, \epsilon_{2}=2.9$, dielectric constant of the particles 11 , volume fraction of the particles 0.26 , appraent density $0.74 \mathrm{~g} / \mathrm{ml}$, and density of the corn oil $0.92 \mathrm{~g} / \mathrm{ml}$. Based on these parameters, it is straightforward to obtain $\alpha=1.5 \times 10^{-7} \mathrm{~cm}^{3}, \rho=0.228 \mathrm{~g} / \mathrm{cm}^{3}$, and $m=1.02 \times 10^{-7} \mathrm{~g}$. If take $g_{L}=1 / 8, \chi=83.7 \beta$. Further, as $\beta=2.1 \times 10^{-10} \mathrm{~Pa}^{-1}, \chi=1.76 \times 10^{-9}(\mathrm{~V} / \mathrm{cm})^{-2}$. To one's interest, Eq. (21) has exactly bridged the mechanical properties and nonlinear dielectric properties of the linear ER fluids. In other words, the mechanical properties give rise to the nonlinear dielectric responses (third-order nonlinear susceptibilities) of the linear ER fluids.

In what follows, we would like to show the correctness of the present theory by comparing with a different statistical method. First, let us derive the increase of the density $\Delta \rho$ due to electrostriction, based on $(\partial V / \partial\langle\mathbf{E}\rangle)_{T, p}$. Let us start from

$$
\Delta \rho=\iint_{0}^{\langle\mathbf{E}\rangle} f(d)\left(\frac{\partial \rho(d)}{\partial\langle\mathbf{E}\rangle}\right)_{T, p} \mathrm{~d}\langle\mathbf{E}\rangle \mathrm{d} d .
$$

To this end, we obtain

$$
\Delta \rho=\frac{1}{8 \pi} \int f(d)\langle\mathbf{E}\rangle^{2} \beta \rho(d)^{2}\left(\frac{\partial \epsilon_{e}}{\partial \rho(d)}\right)_{T} \mathrm{~d} d .
$$


Again, in the expression for $\Delta \rho$ terms in powers of $\langle\mathbf{E}\rangle$ higher than the second have been neglected. For a monodisperse case, Eq. (24) reduces to

$$
\Delta \rho=\frac{1}{8 \pi}\langle\mathbf{E}\rangle^{2} \beta \rho^{2}\left(\frac{\partial \epsilon_{e}}{\partial \rho}\right)_{T} .
$$

Let us assume there is an ideal gas inside the capacitor. In this case, the compressibility is given by

$$
\beta=\frac{M}{\rho R T},
$$

where $M$ is the molecular weight, and $R$ the molar gas constant. For the ideal gas (monodisperse case), setting $g_{L}=1 / 3$ to the above Clausius-Mossotti equation [Eq. (22)] yields

$$
\frac{\epsilon_{e}-1}{\epsilon_{e}+2}=\frac{4 \pi}{3} \frac{\rho}{m} \alpha
$$

In view of $\epsilon_{e}-1 \ll 1$ for ideal gases, we obtain

$$
\left(\frac{\partial \epsilon_{e}}{\partial \rho}\right)_{T}=\frac{4 \pi}{m} \alpha
$$

and hence the desired results for $\Delta \rho$,

$$
\Delta \rho=\frac{\langle\mathbf{E}\rangle^{2} \rho \alpha}{2 k_{B} T}
$$

This equation can also be achieved by using a statistical method. According to Boltzmann's distribution law, the number of moles per $\mathrm{cm}^{3}$ of the gas at a point with field strength $\langle\mathbf{E}\rangle$ is given by

$$
N=N^{\prime} \exp \left(-\frac{W}{k_{B} T}\right)
$$

where $W$ denotes the average value of the work required to bring a molecule into the field $\langle\mathbf{E}\rangle$, and $N^{\prime}$ the number of moles per $\mathrm{cm}^{3}$ of the gas at a point in the absence of field. It is straightforward to obtain

$$
\Delta \rho=M\left(N-N^{\prime}\right)=\frac{\langle\mathbf{E}\rangle^{2} \rho \alpha}{2 k_{B} T},
$$


which is exactly the same as Eq. (29). Again, the terms in higher powers of $\langle\mathbf{E}\rangle$ than the second have been neglected.

To sum up, by using thermodynamics we have presented a first-principles approach to the derivation of the effective third-order nonlinear susceptibility [Eq. (21)] of the linear ER fluids under the influence of electrostriction, which is of about the same order of magnitude as the compressibility of the ER fluid at constant pressure. Our approach has been demonstrated in excellent agreement with an alternative statistical method.

The aim of the present paper is to exploit electrostriction in a linear ER fluid in order to generate a nonlinear dielectric response. The proposed mechanism works for dc electric fields. It should also be expected to work for ac fields with frequency $\nu$ if the size of the sample is not greater than $c_{s} / \nu$, where $c_{s}$ is the sound velocity. In this connection, $\nu$ can be up to $\mathrm{kHz}$ or so. Otherwise the required mass density oscillations will not be able to keep up with the rapid changes in the electric field.

The theory described in this paper can be used to study any colloidal suspensions like magnetorheological fluids [18], ferrofluids [19], et al. Since there exist permanent magnetic dipole moments inside the magnetorheological fluids and ferrofluids, the derivation of the effective linear permeability can still be done by using the present anisotropic ClausiusMossotti equation, in which, however, the terms of permanent magnetic moments should be added accordingly. In a word, we have shown theoretically that the linear ER fluids under the influence of the electrostriction effect can serve as a new nonlinear dielectric material.

\section{ACKNOWLEDGMENTS}

This work was in part supported by the Alexander von Humboldt Foundation of Germany. The author acknowledges Professor K. W. Yu's fruitful discussions. 


\section{REFERENCES}

[1] See, for example, P. W. Smith, Philos. Trans. R. Soc. London A 313, 349 (1984); Nonlinear Photonics, edited by H. M. Gibbs, G. Khitrova, and N. Peyghambarian (SpringerVerlag, New York, 1990); G. I. Stegeman, in Contemporary Nonlinear Optics, edited by G. P. Agrawal and R. W. Boyd (Academic Press, Boston, 1992).

[2] See, for example, D. C. Dodenberger, J. R. Heflin, and A. F. Garito, Nature (London) 359, 309 (1992); D. J. Bergman and D. Stroud, Solid State Phys. 46, 147 (1992); G. L. Fischer, R. W. Boyd, R. J. Gehr, S. A. Jenekhe, J. A. Osaheni, J. E. Sipe and L. A. Weller-Brophy, Phys. Rev. Lett. 74, 1871 (1995).

[3] W. M. Winslow, J. Appl. Phys.20, 1137 (1949).

[4] R. Tao, J. M. Sun, Phys. Rev. Lett. 67, 398 (1991).

[5] T. C. Halsey, Science 258, 761 (1992).

[6] For example, see Electrorheological Fluids, edited by R. Tao (World Scientific, Singapore, 1992); Electrorheological Fluids, edited by R. Tao and G. D. Roy (World Scientific, Singapore, 1994); Electro-Rheological Fluids, Magneto-Rheological Suspensions and Associated Technology, edited by W. A. Bullough (World Scientific, Singapore, 1996); Electrorheological Fluids and Magetorheological Suspensions, edited by G. Bossis (World Scientific, Singapore, 2001).

[7] W. J. Wen, X. X. Huang, S. H. Yang, K. Q. Lu, and P. Sheng, Nature Materials 2, 727 (2003).

[8] C. J.F. Böttcher, Theory of electric polarization, second edition, (Elsevier, Amsterdam, 1993), Vol. 1.

[9] G. A. Zimmerli, R. A. Wilkinson, R. A. Ferrell, and M. R. Moldover, Phys. Rev. Lett. 82, $5253(1999)$. 
[10] W. Lehmann, H. Skupin, C. Tolksdorf, E. Gebhard, R. Zentel, P. Kruger, M. Losche, F. Kremer, Nature (London) 410, 447 (2001).

[11] J. Y. Li, Phys. Rev. Lett. 90, 217601 (2003).

[12] G. H. Kim and Y. M. shkel, J. Intel. Mat. Syst. Str. 13, 479 (2002).

[13] D. Stroud and P. M. Hui, Phys. Rev. B 37, 8719 (1988).

[14] C. Park and R. E. Robertson, Mat. Sci. Eng. A-Struct. 257, 295 (1998); A. Kawai, K. Uchida, and F. Ikazaki, in Electrorheological Fluids and Magetorheological Suspensions, edited by G. Bossis (World Scientific, Singapore, 2001), pp. 626-632.

[15] J. E. Martin, R. A. Anderson, and C. P. Tigges, J. Chem. Phys. 108, 3765 (1998); ibid, 108, 7887 (1998).

[16] J. P. Huang, J. T. K. Wan, C. K. Lo, and K. W. Yu, Phys. Rev. E 64, 061505(R) (2001).

[17] D. J. Klingenberg, F. V. Swol, and C. F. Zukoski, J. Chem. Phys. 94, 6170 (1991)

[18] V. I. Kordonsky and Z. P. Shulman, in Electrorheological Fluids, edited by J. D. Carlson, A. F. Sprecher, and H. Conrad (Technomic Publishing, Lancaster, Basel, 1991), pp. 437444 .

[19] R. E. Rosensweig, Ferrohydrodynamics (Cambridge Univ. Press, Cambridge, 1985). 